\title{
Reseña
}

\section{León, M. (2014): The transformation of care in European Societies, New York, Palgrave Macmillan. 346 págs.}

Son numerosos los escritos centrados en reflexionar críticamente acerca de los fundamentos filosóficos y éticos del "cuidado", sobre los que se han asentado las sociedades democráticas en Europa en el último siglo (Tronto, 1993; Gilligan, 1982; Held, 2006), pero son más bien escasos los estudios que se han adentrado en los fundamentos teóricos y empíricos del significado de la transformación del "cuidado" en las sociedades europeas desde un enfoque sociológico (Daly y Lewis, 2000). Este es precisamente el reto que se propone la doctora Margarita León, reuniendo en este volumen a los más prestigiosos y acreditados investigadores en esta temática en Europa.

En la primera parte del volumen se contextualizan las transformaciones que ha experimentado el cuidado en Europa. Así en el primer capítulo, León, Ranci y Rostgaard reflexionan sobre el concepto de cuidado en perspectiva histórica planteando el debate en torno al paradigma universalista aplicado a las políticas de cuidado en los diferentes contextos nacionales. En el capítulo segundo de este bloque, los autores León, Pavollini y Rostgaard introducen la perspectiva analítica con el fin de obtener resultados empíricos sobre las diferencias existentes en las políticas sociales del cuidado en los distintos países europeos a partir de los datos procedentes de la European Social Survey y la OCDE, subrayando la diferente implicación de los gobiernos en la atención dedicada a los servicios de cuidado. En el capítulo tres, Van Hooren explora las conexiones existentes entre los flujos migratorios y la arquitectura institucional de los "regímenes de cuidado" con el objetivo último de presentar evidencias empíricas sobre la variación existente en el diseño institucional y la integración de los inmigrantes en el mercado laboral como cuidadores, como consecuencia de la globalización y del envejecimiento de la población europea, cada vez más demandante de cuidadores de la tercera edad. Se centra en estudios de caso en Austria, Reino Unido, Polonia e Italia. En el capítulo cuatro, Pfau Effinger incide en la necesidad de considerar las diferencias culturales entre países para explicar las diferencias observadas en las políticas sociales de cuidado. La autora destaca las relaciones existentes entre los factores socioeconómicos, institucionales y culturales para explicar las contradicciones existentes entre las preferencias individuales y en la gestión pública y privada del cuidado realizada por los Estados y las familias. Para ello utiliza la noción de "gender arrangement", con el fin de explicar las diferentes tipologías de regímenes de bienestar en relación con las políticas de cuidado, el empleo femenino y el papel de las familias. Chung y Meuleman en el capítulo cinco exploran la relación entre el nivel macro y micro a través del análisis de las preferencias, actitudes y comportamientos relativos a cómo deber ser gestionado y 
organizado el cuidado de los menores, con el fin último de reflexionar si tales actitudes son coincidentes con el modelo de Estado de bienestar presente en cada país. Albertini por su parte en el capítulo seis presenta un análisis de los diferentes formatos de cuidado formal e informal a la tercera edad en Austria, Alemania, Dinamarca, Italia, España y Polonia con datos procedentes de la Encuesta de Salud, envejecimiento y retiro en Europa (SHARE). A partir de este análisis empírico Albertini trata de buscar algún tipo de asociación entre las políticas de cuidado a la tercera edad y la arquitectura de los modernos Estados de bienestar en los diferentes contextos analizados.

La segunda parte del libro se basa en estudios comparados de casos nacionales que ejemplifican diferentes tradiciones institucionales y familiares del cuidado con el fin de facilitar de esta forma la comparación, ya que de otra forma sería prácticamente imposible dada la inexistencia de datos comparables. En el capítulo siete Österle analiza las implicaciones de la trasnacionalización del cuidado de los mayores dependientes en Austria. Su estudio se basa en explicar cómo los trabajadores inmigrantes han transformado el escenario de la sanidad pública y de la gestión privada del cuidado en Austria, convirtiéndose este colectivo en un pilar fundamental del sistema de cuidado y del Estado de bienestar austriaco. Rostgaard en el capítulo ocho se adentra en la exploración del modelo público danés de cuidado para los dependientes (niños y tercera edad). Reflexiona sobre como los cambios producidos en la forma de gestionar esos servicios podría estar cambiando el significado de la política social en este país, teniendo un claro efecto sobre el acceso y la calidad del cuidado en detrimento del principio del universalismo, característico de las políticas sociales de los países del norte de Europa. En el capítulo nueve Shober se centra en analizar los cambios que se han producido en la educación pre-escolar y las políticas de cuidado destinadas a los niños menores de tres años en Alemania desde finales de la década de los noventa hasta la actualidad. A partir de datos procedentes de diferentes fuentes Shober evalúa hasta qué punto los cambios experimentados en los permisos parentales y en los programas de gasto destinados a las familias con hijos suponen una mayor calidad en los servicios de cuidado y educación destinados a los niños en edad pre-escolar. En el capítulo diez Ranci y Sabatinelli analizan desde una perspectiva institucional la evolución y las transformaciones que han experimentado los servicios de cuidado a la tercera edad y a los niños menores de tres años en Italia, como consecuencia de la intensificación de los procesos migratorios. Los autores reflexionan sobre la calidad de estos servicios de cuidado, tanto formales e informales, que se articulan en torno al eje de la privatización y familiarización que caracteriza a los regímenes de bienestar del sur de Europa. A continuación, en el capítulo once, Perek-Bialas y Raclaw analizan críticamente el estado de las políticas sociales de cuidados destinadas a la tercera edad en Polonia. Los autores incorporan la perspectiva histórica para proporcionar un modelo analítico explicativo de los cambios experimentados en la política social destinada a la tercera edad como consecuencia de las interacciones cambiantes que regulan la relación entre el Estado de bienestar, la familia, el mercado laboral y los procesos migratorios. En el capítulo doce Ibánez y León analizan el estado de los servicios de educación pre-escolar en España en perspectiva histórica atendiendo a la calidad de los servicios y a las condiciones laborales de los profesionales que prestan estos servicios en el marco de las políticas familiares 
desarrolladas por el Estado de bienestar español. Realizan este análisis a partir de los datos procedentes de fuentes secundarias, entrevistas en profundidad y análisis de dos casos de servicios públicos de atención a los niños en edad pre-escolar. Por otra parte Shutes analiza en el capítulo trece, el estado de los servicios a la tercera edad en el Reino Unido focalizando la atención en la división existente en la provisión de estos servicios en función de la condición de ciudadano nacional o emigrante que presta y recibe estos servicios. Una de las conclusiones es que los inmigrantes son empleados en este sector de cuidados, generalmente privatizado y con precarias condiciones laborales. Para concluir, el último capítulo, León sintetiza las transformaciones que han experimentado en Europa los servicios de cuidados y las respuestas institucionales diferenciadas que se están dando a las nuevas necesidades de cuidado derivadas del un nuevo escenario demográfico, laboral y familiar desde los diferentes arquetipos institucionales de los regímenes de bienestar en términos de cobertura, calidad de los servicios y condiciones laborales de los empleados en dichos sectores.

En definitiva este libro supone una aportación novedosa en términos teóricos y empíricos a los escasos análisis comprados existentes en torno al concepto de cuidado (Lewis, 2006; Daly y Lewis, 2000) y a la prestación de estos servicios de cuidados destinados a los niños menores de tres años y a la tercera edad en los distintos regímenes de bienestar europeos. Este libro añade además nuevos datos y reflexiones a las escasas fuentes disponibles para evaluar y comparar las respuestas institucionales ofrecidas por los distintos Estados de bienestar a las crecientes necesidades de cuidado a los dependientes en los distintos contextos nacionales europeos.

\author{
Almudena Moreno Mínguez \\ Universidad de Valladolid \\ Departamento de Sociología y Trabajo Social \\ almudena@soc.uva.es
}

\title{
Bibliografía
}

Daly, M. y J. Lewis (2000): "The concept of social care and the analysis of contemporary welfare States", British Journal of Sociology, 51 (2), pp. 281-298.

Gilligan, C. (1982). A different voice: Psychological theory and women's development, Massachussets, Harvard University Press.

Held, V. (2006): The ethics of care: personal, political and global, Oxford, Oxford University Press.

Lewis, J. (2006): "Men, women, work, care and policies", Journal of European Social Policy, 16 (4), pp. 387-392.

Tronto, J. (1993): Moral Boundaries: A Political argument for an ethic of care, New York, Routledge. 